\title{
Implementação de uma rede IoT LoRaWAN para integração com o núcleo 5G
}

\author{
Cláudio da Silva P. Júnior ${ }^{1}$, Rogério S. Silva ${ }^{1,2}$, Samuel Wanberg ${ }^{1}$, \\ Kleber V. Cardoso ${ }^{1}$ e Antonio Oliveira-Jr ${ }^{1,3}$ \\ ${ }^{1}$ Instituto de Informática (INF) - Universidade Federal do Goiás (UFG) \\ Alameda Palmeiras, Quadra D, Câmpus Samambaia \\ 74.69-900 - Goiânia - GO - Brazil \\ ${ }^{2}$ Instituto Federal de Goiás (IFG) - Núcleo de Estudos aplicados \\ a Redes de computadores e Sistemas distribuídos (NumbERS) \\ Avenida Universitária, $\mathrm{s} / \mathrm{n}^{\circ}$, Vale das Goiabeiras \\ 75.400-000 - Goiânia - GO - Brazil \\ ${ }^{3}$ Fraunhofer Portugal AICOS \\ Porto, Portugal \\ \{claudiospj, samuelwanberg\}@gmail.com, rogerio.sousa@ifg.edu.br \\ \{kleber, antonio\}@inf.ufg.br
}

\begin{abstract}
Integration of Internet of Things (IoT) LoRaWAN networks with the $5 G$ system aims to maximize the possibilities of expanding wireless network connectivity coverage in order to minimize existing gaps in Brazil due to its territorial extension, which causes a low level of connectivity in remote areas. This work presents an implementation of a LoRaWAN IoT network using ChirpStack for integration with $5 G$ core. Use cases are presented using a simulated environment, a real environment and a complete environment that adopts grafana to expose metrics in a dashboard.
\end{abstract}

Resumo. Integração de redes IoT LoRaWAN com o sistema $5 G$ visa maximizar as possibilidades de expansão de cobertura da conectividade das redes sem fio a fim de minimizar lacunas existentes no Brasil em decorrência da sua vasta extensão territorial que causa um baixo nivel de conectividades de áreas remotas. Este trabalho apresenta uma implementação de uma rede IoT LoRaWAN utilizando o ChirpStack para integração com o núcleo 5G. São apresentados casos de uso de um ambiente simulado, ambiente real e um ambiente completo utilizando o grafana para métricas em dashboard.

\section{Introdução}

Um aumento expressivo no número de dispositivos conectados, estima-se bilhões, está demandando uma transformação nos sistemas de comunicação móveis atuais na chamada Internet das Coisas (IoT) [Kodheli et al. 2021, Rumana et al. 2017]. A evolução das gerações da comunicação móvel celular, i.e. $4 \mathrm{G}, 3 \mathrm{G}$, e anteriores, focaram principalmente em aumentar a taxa de transferência de dados, porém, para as novas demandas da rede de Quinta Geração (5G), isto não é mais suficiente. O 5G está quebrando este paradigma, e fornecerá conexões de rádio e conectividade de rede ponta 
a ponta que permitirá não apenas Banda larga móvel aprimorada (Enhanced Mobile Broadband (EMBB)), mas também menor latência e maior confiabilidade através das comunicações de baixa latência ultra confiáveis (Ultra Reliable Low Latency Communications (URLLC)), e conectividade massiva de dispositivos (Massive Machine Type Communications (MMTC)) [Pope 2018, Hedman et al. 2019]. As redes 5G realizarão uma transformação significativa na economia e no modo como a sociedade lida com tecnologia.

As redes $5 \mathrm{G}$ e as redes futuras possibilitarão acesso a dispositivos inteligentes em todo o globo terrestre, incluindo espaço, ar, terra e mar [Kodheli et al. 2021]. Para alcançar cobertura global, com alcance não somente às áreas tipicamente urbanas e com alta densidade populacional, mas também a locais remotos, faz-se necessário ampliar significativamente a rede. Todavia, a implantação de infraestrutura por parte das operadoras demanda altíssimo investimento em compras de produtos (Capital Expenditure (CAPEX)) e em zonas de baixa densidade populacional o retorno financeiro (Return On Investment (ROI)) é naturalmente baixo [Mendes et al. 2020].

De um outro prisma, para além das necessidades de aplicações EMBB, as zonas remotas também demandam aplicações URLLC, por exemplo, para verificação de pragas por meio de Veículos Aéreos Não Tripulados (VANT), e aplicações MMTC, por exemplo, para o sensoriamento de barragens. No contexto do MMTC, há alternativas de integração que podem diminuir o CAPEX na implantação dessa nova infraestrutura. Para isto, envolvem a integração de outras tecnologias de acesso de rádio (Radio Access Technology (RAT)) que adotam espectros de frequência não-licenciados, por exemplo, SigFox [Sigfox 2021], Long Range (LoRa) [ChirpStack 2019], além dos de frequência licenciada, por exemplo, NB-IoT e LTE-M [Mikhaylov et al. 2018].

O desenvolvimento do sistema 5G (5G System (5GS)) é padronizado principalmente pela 3rd Generation Partnership Project (3GPP), que atua definindo e mantendo padrões técnicos e normas. Esta padronização tem como objetivo alcançar os requisitos para a implantação da rede de telecomunicações móveis em todo o mundo [Pope 2018]. A rede de acesso 5G usando New Radio (NR)-Stand-Alone Architecture (SA) e o núcleo da rede $5 \mathrm{G}$ (5G Core (5GC)) estão padronizados pela $3 \mathrm{GPP}$ e o acesso pode ser realizado de dois modos, denominados acesso $3 \mathrm{GPP}$ e acesso não-3GPP. No acesso $3 \mathrm{GPP}$, um User Equipment (UE) conecta, de forma confiável, diretamente ao núcleo da rede 5G. Entretanto, existem redes de acesso não-3GPP, nos quais a operadora móvel não confia no ponto de acesso ao qual o UE está conectado, pode ser uma rede local sem fio (por exemplo, de uma casa), ou uma rede IoT (por exemplo, sensores e dispositivos IoT de uma indústria ou propriedade rural). No acesso não-3GPP, padronizado no Lançamento 15 do 5G, o UE canaliza todo o tráfego para um gateway na rede, o qual é confiável para a operadora móvel [Silva et al. 2021].

Várias RAT estão disponíveis atualmente, dentre elas, as Low-power wide area networks (LPWAN) se destacam por fornecer acesso a longas distâncias e com alta eficiência energética [Mikhaylov et al. 2018]. LPWANs buscam cobrir a lacuna entre as tecnologias celulares tradicionais e os requisitos atuais de mMTC [Navarro-Ortiz et al. 2018]. Long Range Wide Area Network (LoRaWAN) se posiciona em destaque entre as tecnologias LPWANs devido às suas excelentes características, como baixíssimo custo (para dispositivos finais e infraestru- 
tura), baixíssimo consumo de energia, alcance muito longo, comunicação bidirecional e alta segurança. [Rumana et al. 2017]. LoRaWAN é um protocolo que define a camada de acesso ao meio (Media Access Control (MAC)), a camada física inferior é definida pela LoRa, que se baseia em técnicas de modulação de espectro de propagação derivadas da tecnologia Chirp Spread Spectrum (CSS) [Semtech 2021]. As especificações LoRa/LoRaWAN são desenvolvidas e mantidas pela associação LoraAlliance ${ }^{\circledR}[$ LoRa 2021] .

O presente trabalho compõe um conjunto de aplicações para o acesso não confiável de dispositivos IoT não-3GPP ao 5GS. Para definir um ambiente de testes e validação desses acessos são necessários, um 5GS funcional que contempla, rede de acesso, transporte e núcleo, uma rede de dispositivos IoT, gateways para a integração desses dispositivos não-3GPP ao 5GC via função de rede Non-3GPP InterWorking Function (N3IWF) e de servidores de rede e de aplicações LoRaWAN. Alguns sistemas 5G estão disponíveis atualmente, entre eles a iniciativa my5G [Cardoso et al. 2021b] e o núcleo 5G, my5G-core [Cardoso et al. 2021a]. Nesse contexto, propõe-se o desenvolvimento de um ambiente com dispositivos LoRa reais e virtuais, operando conjuntamente e integrados ao ChirpStack open-source LoRaWAN Network Server (CLNS) com o objetivo de gerar uma massa de dados de teste para exercitar um gateway IoT definido por software (IoTSDGw), além de aplicações para o acesso não-3GPP ao núcleo da rede 5G por meio da função de rede virtualizada (Virtualized Network Function (VNF)) N3IWF.

Para além dessa introdução, o trabalho está organizado como se segue: na seção 2 são apresentados os principais conceitos relacionados ao trabalho, na seção 3 é apresentada a proposta de trabalho, na seção 4 demonstra-se os resultados obtidos e finalmente na seção 5 apresenta-se a conclusão e os trabalhos futuros.

\section{Fundamentos}

Segundo o 3GPP [3GPP 2020], o sistema 5G (5GS) está sendo desenvolvido inicialmente utilizando a infraestrutura legada das gerações anteriores. Deste modo, desenvolvido como uma arquitetura não-independente (Non Stand-Alone Architecture (NSA)), possibilitou às operadoras de redes móveis (Mobile Network Operator (MNO)) aproveitar a infraestrutura já existente das redes de transporte e núcleo para ofertar serviços $5 \mathrm{G}$, com foco inicial em conectividade de alta velocidade (EMBB) [Hedman et al. 2019]. Todavia o desenvolvimento dos novos lançamentos, partindo do release 15 [Pope 2018] tiveram ênfase em uma arquitetura totalmente independente, denominada (Stand-Alone Architecture (SA)). O 5G-SA está sendo projetado para incluir uma nova rede de acesso ( $R a$ dio Access Network (RAN)), uma nova rede de transporte (Transport Network (TN)) e uma nova rede de núcleo 5G (5G Core Network (5GCN)). A SA 5G é uma arquitetura nativa da nuvem (Cloud-Native Architecture (CNA)), totalmente virtualizada e que apresenta novas maneiras de desenvolver, implantar e gerenciar serviços. A CNA inclui conceitos de microsserviços e interfaces baseadas em serviços (Service-Based Interface (SBI)) [Hedman et al. 2019].

Uma das principais mudanças que o 3GPP propôs para a arquitetura da rede 5G foi o conceito de arquitetura baseada em serviços (Service Based Architecture (SBA)). Na SBA, as funções da rede (Network Functions (NFs)) não são acessadas através de conexões ponto a ponto, mas sim como interfaces de serviço. Neste con- 
texto, as NFs atuam como produtoras ou consumidoras, assim como na arquitetura orientada a serviços (Service-Oriented Architecture (SOA)) oriunda da engenharia de software [Hedman et al. 2019] e acessíveis por meio de interfaces de programação de aplicações (Application Program Interface (API)) [Hedman et al. 2019, Pope 2018]. Outras recentes tecnologias disruptivas, tais como, Redes definidas por software (Software Defined Networks - SDN), Virtualização das Funções de Redes (Network Function Virtualization - NFV), Computação em nuvem (Cloud Computing) e Conteinerização também compõem o rol utilizado para o desenvolvimento do 5GS. Desta forma, é possível observar que a softwarização (i.e., a adoção de funcionalidades desenvolvidas em software em vez de integradas ao hardware) orienta o desenvolvimento das redes de comunicação, e é o caminho natural para a sua evolução [Silva et al. 2021].

Long Range (LoRa) é uma tecnologia de rádio transmissão LPWAN, criada pela empresa SemTech [Semtech 2021] que tem sido muito adotada para soluções IoT de larga escala. LoRa possibilita uma comunicação de longa distância, em geral 3-5 km em regiões urbanas e $16 \mathrm{~km}$ em regiões com poucos obstáculos [Raza et al. 2017], apresenta um consumo de energia extremamente baixo, possibilitando aos dispositivos operarem por longos períodos de tempo (acima de 10 anos), sem precisar sofrer recarga de energia ou troca da fonte de energia.

O LoRaWAN é o protocolo que define a arquitetura do sistema, bem como os parâmetros de comunicação usando a tecnologia LoRa promovido pela LoRa Alliance [LoRa 2021]. O padrão implementa todos os detalhes de funcionamento da rede, segurança, criptografias, qualidade de serviço (QoS), ajustes de potência e frequência visando maximizar a duração da bateria dos módulos, redução de colisões de pacotes, e as categorias de dispositivos e aplicações tanto do lado do dispositivo quanto do servidor [ChirpStack 2019]. Atualmente a cobertura da rede LoRaWAN alcança 162 países, segundo o LoRa Alliance ${ }^{\circledR} 2020$ Annual Report [LoRa 2021] a arquitetura é dividida em quatro partes: os dispositivos, gateways, Network Server e Application Server. Os dispositivos são os nós finais que transmitem diretamente para todos os gateways dentro do alcance, usando LoRa. Os gateways LoRa se conectam à internet por meio do protocolo IP e transmitem os dados recebidos dos sensores LoRa para um servidor de rede. Os servidores de rede se conectam aos gateways, e em seguida, roteia os pacotes recebidos para um aplicativo. Os servidores de rede podem ser usados para comunicação de uplink (ou seja, sensor para aplicativo) ou downlink (aplicativo para sensor). O servidor de aplicações é responsável pelo "inventário" de dispositivos, pelo tratamento das solicitações de conexão e pelo tratamento e criptografia dos payloads dos aplicativos [LoRa 2021, ChirpStack 2019].

O protocolo Message Queuing Telemetry Transport (MQTT) foi desenvolvido pela IBM no final dos anos 90 com o intuito de vincular sensores de pipelines de petróleo a satélites. Esse protocolo foi criado para ter suporte a mensagens assíncronas, além de ser desacoplado entre o emissor e o receptor da mensagem tanto em referência ao espaço quanto ao tempo. MQTT é um protocolo de comunicação do tipo Publish-Subscribe (PUB-SUB) (com um servidor central, chamado broker), sendo largamente utilizado nos projetos e aplicações em Internet das Coisas, o que o torna escalável e adequado para ambientes de redes não confiáveis como LoRaWAN. Em 2014, tornou-se oficialmente um padrão aberto da OASIS e em 2019 foi lançado a versão 5 [OASIS 2021]. 
Com o advento das redes 5G e de Sexta Geração (6G) surge a necessidade de integração das tecnologias de comunicação sem fio IoT com o 5GCN. Nesse interim, os principais desafios envolvem a implementação dos padrões da 3GPP, especialmente no que tange aos Lançamentos 15 e posteriores no âmbito da definição e desenvolvimento de uma solução para contemplar tecnologias não-licenciadas não3GPP [Pope 2018, Firmin and Kymalaine 2020]. Atualmente, as implementações de código abertas do 5GS-SA, especificamente do 5GCN estão em estágio de desenvolvimento e apesar de especificadas pelo 3GPP, não foram encontrados na literatura vestígios do desenvolvimento de integrações para IoT não confiáveis não-3GPP com o 5GCN-SA. A próxima seção apresenta o desenvolvimento de uma rede LoRaWAN para integração de dispositivos LoRa/LoRaWAN com o núcleo da rede 5G através da N3IWF.

\section{Implementação de uma rede LoRaWAN utilizando ChirpStack}

O presente trabalho realizou a implementação de uma rede LoRa/LoRaWAN utilizando o software CLNS [ChirpStack 2019]. O CLNS é composto por um conjunto de componentes, são eles, (i) o Gateway Bridge responsável por receber os pacotes enviados por um gateway LoRa, (ii) o Network Server responsável pelo manuseio dos pacotes e (iii) o Application Server que fica a cargo de inventariar os dispositivos, fazer o tratamento de integrações, descriptografias, entre outros. Para a simulação dos dispositivos IoT, foi considerado o projeto LoRa Device Simulator (LDS) [Gómez 2021], desenvolvido especificamente para a plataforma ChirpStack, no entanto, devido aos limites no número de instâncias simuladas pelo LDS não atender às necessidades do projeto, adotou-se o ChirpStack-Simulator [Brocaar 2019] capaz de emular vários dispositivos simultaneamente, além de incorporar recursos para exposição de métricas.

Para validação da proposta foi desenvolvido um mecanismo de coleta de informações e métricas de monitoramento da rede LoRaWAN. Três estudos de caso foram desenvolvidos para validar cenários distintos com dispositivos simulados e reais atuando de forma integrada para coleta e análise dos dados da rede.

O primeiro caso de uso, chamado de "Ambiente Simulado", Figura 1, teve o propósito de gerar um alto volume de tráfego na rede através da ChirpStack open-source LoRaWAN Network Server Stack. O objetivo desse cenário é a posterior análise da capacidade do gateway definido por software para o acesso de dispositivos IoT não-3GPP ao núcleo da rede 5G. Para este fim, um conjunto de 100 mil dispositivos IoT-LoRa virtuais foram gerados, por meio do ChirpStack Simulator.

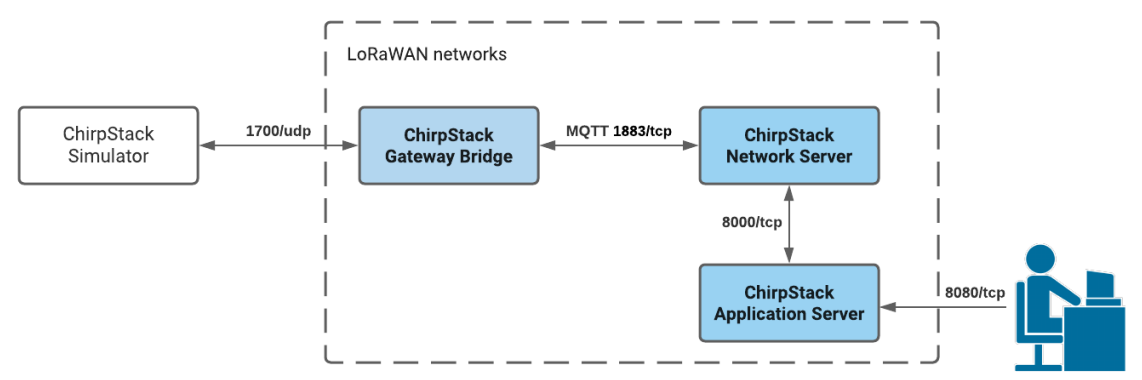

Figure 1. Ambiente Simulado 
No ambiente simulado, Figura 1, os dispositivos virtuais geram tráfego na rede que é encaminhado ao componente ChirpStack Gateway Bridge (CGB) pela porta 1700/udp, responsável pela conversão do protocolo LoRa Packet Forwarded para um protocolo comum, por exemplo, o JavaScript Object Notation (JSON) e Protocol Buffers (ProtoBuf), e em seguida através do protocolo MQTT, na porta 1883/tcp, encaminhá-los ao Chirpstack Network Server (CNS). O CNS é o componente responsável pela rede LoRaWAN de código aberto, o CNS também é responsável pela eliminação da duplicação de frames recebidos pelos gateways LoRa, pela autenticação dos dispositivos, comunicação com o Chirpstack Application Server (CAS), porta 8000/tcp, agendamento de frames de downlink e envio de comandos da rede para os dispositivos. O CAS é então responsável por inventariar os dispositivos que ingressam na rede LoRaWAN, fazer o tratamento de solicitações de ingresso e tratamento de criptografias do payload, oferecer uma interface web amigável na porta 8080/tcp, e de oferecer uma séries de integrações com serviços externos com suporte a gRPC e API REST.

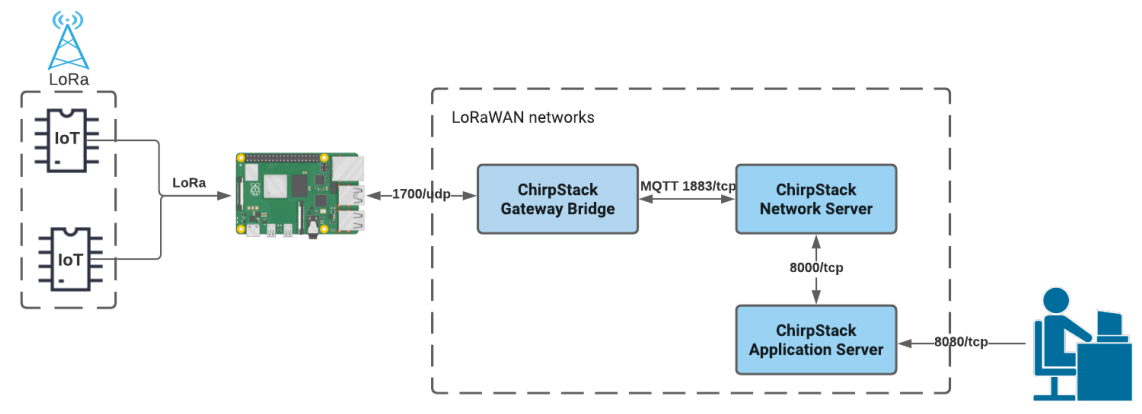

Figure 2. Ambiente Real

O segundo caso de uso, denominado "Ambiente Real", Figura 2, visa observar as características reais na comunicação dos dispositivos e a rede LoRaWAN. Neste cenário foram utilizados dois dispositivos físicos IoT-LoRa (ESP32), um gateway LoRa Pi3 (RFM95W), desenvolvido a partir de um Raspberry PI 3 B+ e um módulo Lora RFM95W. Os dados coletados nos dispositivos são então encaminhados ao componente CGB e o fluxo na rede LoRaWAN é o mesmo relatado anteriormente para o caso de uso "ambiente simulado".

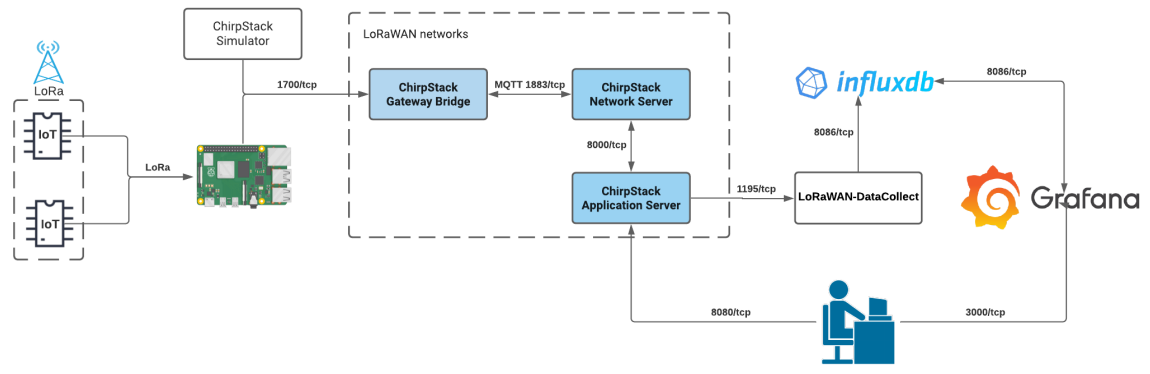

Figure 3. Ambiente Completo

Finalmente, o caso de uso "Ambiente Completo", Figura 3, foi implementado com o objetivo de validar o cenário fim-a-fim, que contempla desde os dispositivos 
IoT (virtuais e reais) até a gestão dos dados obtidos e armazenamento no Banco de Dados de Séries Temporais InfluxDB [Influxdata 2021] e integração com o software Grafana [GrafanaLabs 2021]. Neste contexto, o fluxo a partir da rede LoRaWAN é encaminhado à aplicação LoRa Data Collector (LDC), porta 1195/tcp. A LDC foi desenvolvida em linguagem de programação GO [Google 2021], e é responsável pela captura dos pacotes na rede LoRaWAN e armazenamento dos dados coletados pelo CLNS no InfluxDB, porta 8086/tcp, para serem consumidos pelo software Grafana. O Grafana possibilita a análise dos dados armazenados por meio de dashboards.

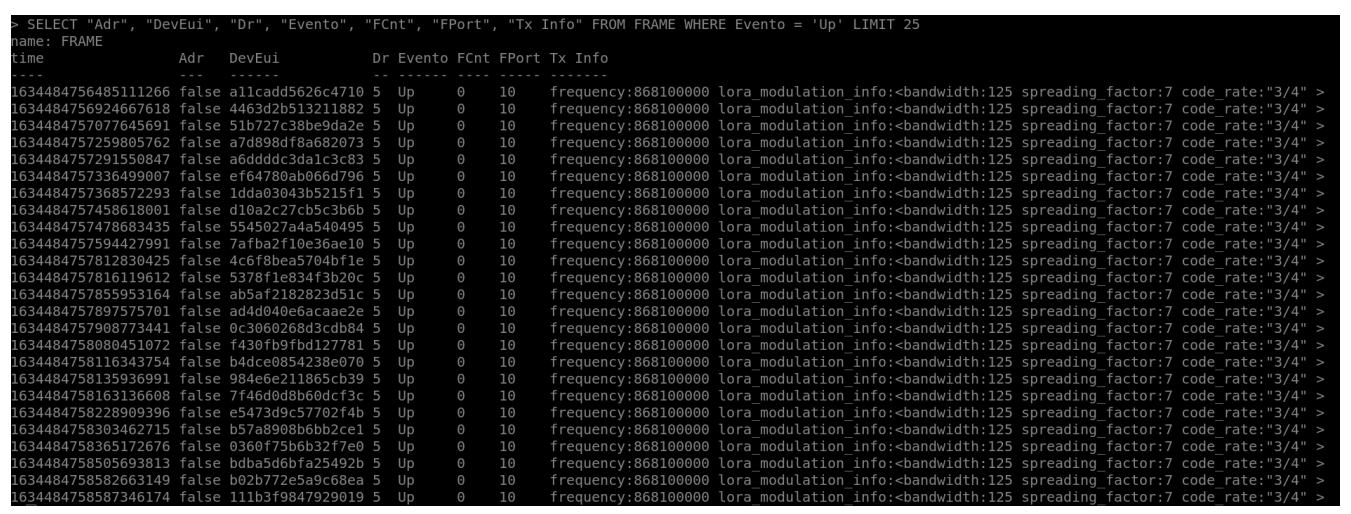

Figure 4. Query no Container InfluxDB

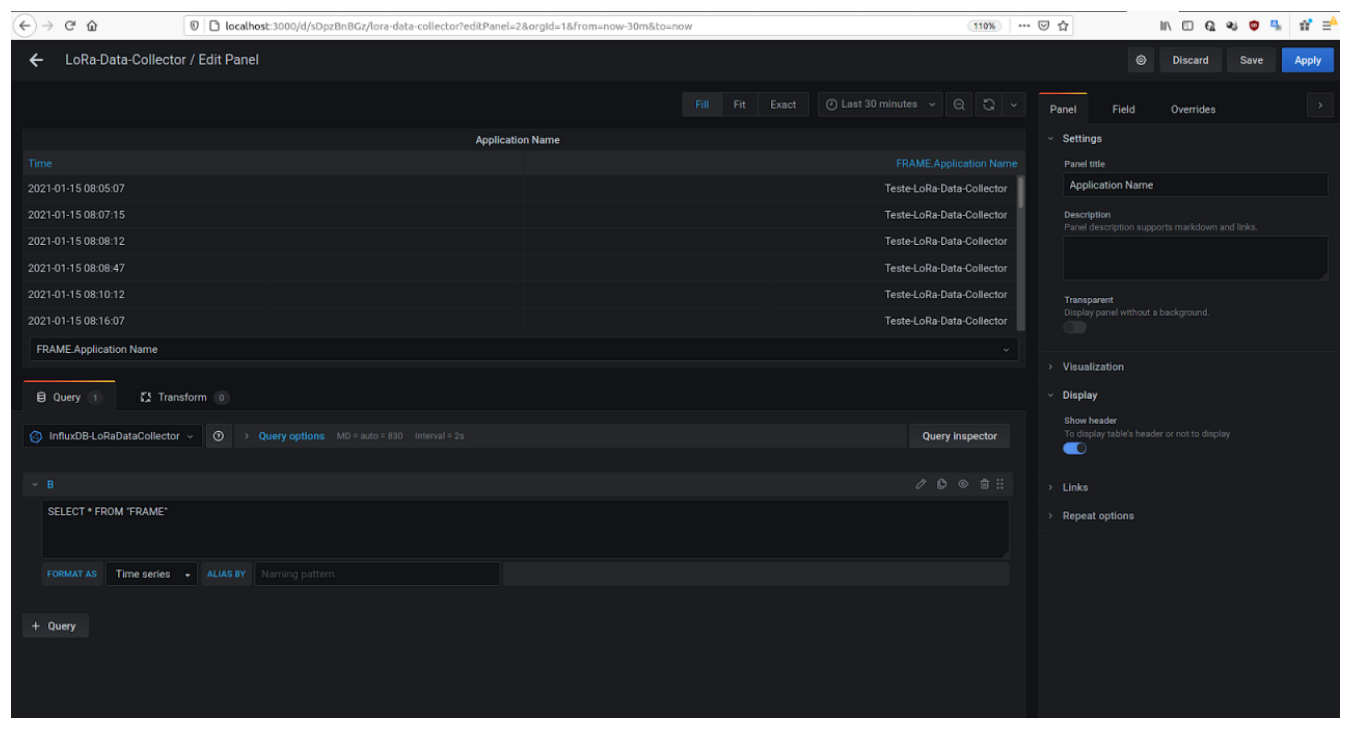

Figure 5. Consultas Grafana

Os dados armazenados no banco de dados Influxdb e as consultas utilizando o Grafana podem ser observadas nas Figuras 4 e 5. Simultaneamente, a ferramenta Grafana gera gráficos para a análise do fluxo de dados na rede (Figura 6).

\section{Resultados}

O experimento contou com 3 cenários, ambos obtiveram êxito em sua execução e alcançaram os resultados esperados. $\mathrm{O}$ ambiente simulado demonstrou a capacidade de geração de um número muito grande de dispositivos, aproximando a simulação 


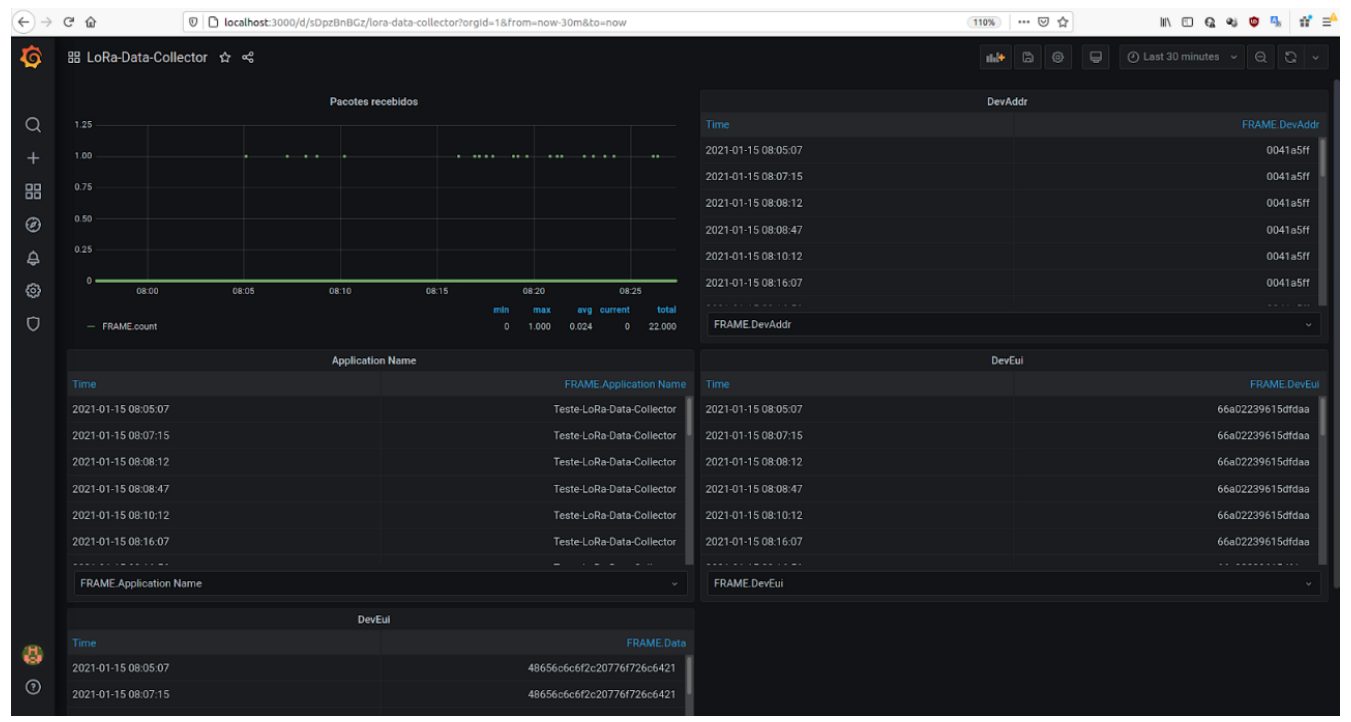

Figure 6. Dados dos Dispositivos loT

de um cenário, por exemplo, de uma cidade inteligente interagindo com os componentes do CLNS. O ambiente real demonstrou a possibilidade de criação de uma rede LoRa/LoRaWAN usando componentes básicos, de fácil desenvolvimento e implantação, seguindo um passo a passo que está disponível no repositório github ${ }^{1}$ desse projeto. $\mathrm{O}$ cenário 3, "Ambiente completo" foi utilizado para a integração com o software IoTSDGw. São apresentados em tempo de execução a integração, entre os dispositivos IoTLoRa (reais e simulados) com os componentes do CLNS. Simultaneamente, os pacotes de dados são capturados pela aplicação LDC e armazenados no banco da dados de séries temporais, InfluxDB, alimentam o dashboard do Grafana.

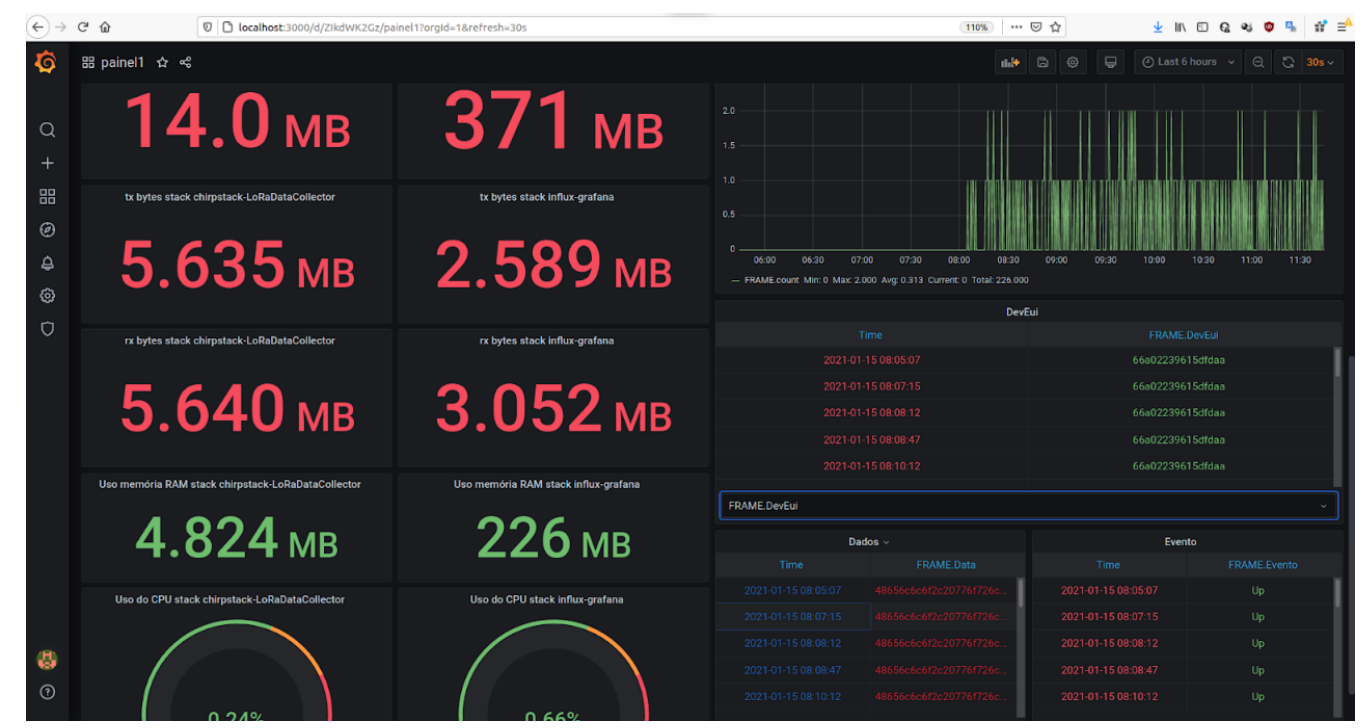

Figure 7. Dashboard de Métricas

O experimento de teste de carga foi realizado simulando até 100 mil dispositivos IoT que ingressam na rede LoRa/LoRaWAN usando o mecanismo Over-the-Air (OTA)

\footnotetext{
${ }^{1}$ https://github.com/LABORA-INF-UFG/LoRa-LoRaWAN-ChirpStack
} 
para fazer o ingresso na rede com o procedimento automatizado de join e envio de mensagens com certa periodicidade. Foram utilizados 5 gateways como porta de entrada dos pacotes enviados pelos dispositivos para a rede LoRa/LoRaWAN. A Figura 7 apresenta os recebimentos dos pacotes com evento $U p$, mensagem enviada pelo dispositivo e recebida pelo Network Server, o uso de disco, memória RAM e CPU pelo software Grafana que consome dados do banco de dados InfluxDB.

\section{Conclusões}

O advento das comunicações móveis $5 \mathrm{G}$ possibilitará o alcance da cobertura de sinal à locais remotos e, desta forma, minimizará as lacunas hoje existentes no Brasil, principalmente nas propriedades rurais. A implantação de redes IoT LPWAN é uma forma de ampliar a cobertura de sinal nestas propriedades de forma a permitir o sensoriamento de dados de lavouras ou de animais e também a atuação nesses ambientes, por exemplo na irrigação das lavouras ou na alimentação de animais, minimizando a intervenção humana e garantindo maior precisão nessas atividades. A proposta apresentada nesse trabalho considera estes cenários, entre outros. A integração de dispositivos IoT não-3GPP à rede 5G é uma maneira de atender a esta demanda. O artigo apresentou a implementação de uma rede IoT LoRaWAN utilizando o CLNS para acesso não-3GPP não confiável com o núcleo 5G. Foram apresentados casos de uso de um ambiente simulado, ambiente real e um ambiente completo para a geração de uma masse da dados para exercitar um IoTSDGw com vistas ao acesso ao 5GCN.

$\mathrm{O}$ experimento de teste de carga chegou a ser realizado simulando até $100 \mathrm{mil}$ dispositivos IoT que ingressam na rede LoRa/LoRaWAN usando o mecanismo Over-theAir (OTA) para fazer o ingresso na rede com o procedimento automatizado de join e envio de mensagens com certa periodicidade. Foram utilizados 5 gateways como porta de entrada dos pacotes enviados pelos dispositivos à rede LoRaWAN.

Têm-se a expectativa da utilização do cenário de ambiente completo para fomentar os testes de validação da ferramenta IoTSDGw. Futuramente este trabalho será expandido para simulação de outros dispositivos IoT não-3GPP, confiáveis e não-confiáveis.

\section{References}

3GPP (2020). 3rd generation partnership project (3gpp). https : / /www . 3gpp . org/.

Brocaar, O. (2019). Chirpstack simulator. https://github.com/brocaar/ chirpstack-simulator.

Cardoso, K. V., Both, C. B., Oliveira-Jr, A. C., Nery, S. W. L., and Silva, R. S. (2021a). my5G-core. https://github. com/my5G/my5G-core.

Cardoso, K. V., Both, C. B., Oliveira-Jr, A. C., Nery, S. W. L., and Silva, R. S. (2021b). my5G Initiative. https://my5g.github. io/.

ChirpStack (2019). Chirpstack open-source lorawan network server. https: / / www . chirpstack.io/.

Firmin, F. and Kymalaine, K. (2020). 3gpp ts24.502 v16.3.0 (2020-03), access to the $3 \mathrm{gpp} 5 \mathrm{~g}$ core network $(5 \mathrm{gcn})$ via non-3gpp access networks. Technical report.

Google (2021). The go project. https: / / golang • org/. 
GrafanaLabs (2021). Grafana stack. https: //grafana.com/.

Gómez, I. (2021). Lora device simulator (lds). https : / / github . com/iegomez / lds.

Hedman, P., Frid, L., Rommer, S., Olsson, M., Sultana, S., and Mulligan, C. (2019). 5G Core Networks: Powering Digitization. Elsevier Science \& Technology.

Influxdata (2021). Influxdb oss v1.8. https://docs.influxdata.com/ influxdb/v1.8/.

Kodheli, O., Lagunas, E., Maturo, N., Sharma, S. K., Shankar, B., Montoya, J. F. M., Duncan, J. C. M., Spano, D., Chatzinotas, S., Kisseleff, S., Querol, J., Lei, L., Vu, T. X., and Goussetis, G. (2021). Satellite communications in the new space era: A survey and future challenges. IEEE Communications Surveys Tutorials, 23(1):70-109.

LoRa (2021). Lora alliance. https: / / lora-alliance.org/.

Mendes, L. L., Moreno, C. S., Marquezini, M. V., Cavalcante, A. M., Neuhaus, P., Seki, J., Aniceto, N. F. T., Karvonen, H., Vidal, I., Valera, F., Barreto, P. A. S. M., Caetano, M. F., Dias, W. D., and Fettweis, G. (2020). Enhanced remote areas communications: The missing scenario for $5 \mathrm{~g}$ and beyond $5 \mathrm{~g}$ networks. IEEE Access, 8:219859-219880.

Mikhaylov, K., Stusek, M., Masek, P., Petrov, V., Petajajarvi, J., Andreev, S., Pokorny, J., Hosek, J., Pouttu, A., and Koucheryavy, Y. (2018). Multi-rat lpwan in smart cities: Trial of lorawan and nb-iot integration. In 2018 IEEE International Conference on Communications (ICC), pages 1-6.

Navarro-Ortiz, J., Sendra, S., Ameigeiras, P., and Lopez-Soler, J. M. (2018). Integration of lorawan and $4 \mathrm{~g} / 5 \mathrm{~g}$ for the industrial internet of things. IEEE Communications Magazine, 56(2):60-67.

OASIS (2021). OASIS Standard - MQTT Version 5.0. https://docs. oasis-open.org/mqtt/mqtt/v5.0/mqtt-v5.0.html.

Pope, M. (2018). System architecture for the 5gs. Technical Report 3GPP TS23.501 V15.2.0, 3rd Generation Partnership Project-3GPP, Sophia Antipolis CEDEX, France. https://www.3gpp.org/ftp/Specs/archive/23_series/23.501/.

Raza, U., Kulkarni, P., and Sooriyabandara, M. (2017). Low power wide area networks: An overview. IEEE Communications Surveys Tutorials, 19(2):855-873.

Rumana, Y., Petäjäjärvi, J., Mikhaylov, K., and Pouttu, A. (2017). On the integration of lorawan with the 5g test network. In 2017 IEEE 28th Annual International Symposium on Personal, Indoor, and Mobile Radio Communications (PIMRC), pages 1-6.

Semtech (2021). What are lora and lorawan? https://lora-developers. semtech.com/library/tech-papers-and-guides/

lora-and-lorawan/.

Sigfox (2021). Sigfox.com. https://www.sigfox.com/en.

Silva, J. P. L., Nery, S. W. L., Silva, R., Oliveira-Jr, A. C., Cardoso, K. V., and Both, C. B. (2021). Entendendo o núcleo $5 \mathrm{~g}$ na prática, através de uma implementação de código aberto. In SBRT 2020: Livro de Minicursos, chapter 1. Instituto Federal de Ensino, Ciência e Tecnologia da Paraíba - IFPB, Florianópolis-SC. 